\title{
La formación tecno-pedagógica de los profesores universitarios de lengua extranjera
}

The technological and pedagogical training of foreign language teachers

\author{
Elizabeth Alvarado-Martínez ${ }^{a}$
}

\begin{abstract}
:
Through this article, different points of view on teacher training in the university field will be addressed, highlighting some aspects of teaching, such as teacher training in ICT, the contributions of ICT in the teaching-learning process in virtual education, educational technology and the role of the university. In addition, the considerations of the educational model proposed by the central administration of the UANL are reviewed, in order to specify some of the problems that university teachers face when carrying out the aforementioned model.
\end{abstract}

Keywords:

ICT, techno-pedagogical training, virtual education

\section{Resumen:}

A través de este artículo, se abordarán diferentes puntos de vista sobre formación docente en el ámbito universitario, resaltando algunos aspectos de la docencia, como la formación docente en las TIC, las aportaciones de las TIC en el proceso de enseñanzaaprendizaje en la educación virtual, la tecnología educativa y el papel de la universidad. Además, se revisan las consideraciones del modelo educativo propuesto por la administración central de la UANL, con el fin de especificar algunas de las problemáticas con las que los docentes universitarios se enfrentan al llevar a cabo el modelo mencionado.

Palabras clave :

Educación virtual, formación tecno-pedagógica, TIC

\section{CONTEXTO DE LA UNIVERSIDAD AUTÓNOMA DE NUEVO LEÓN}

Los sistemas educativos de todo el mundo se enfrentan actualmente al desafío de utilizar las tecnologías de la información y la comunicación (TIC) para proveer a sus alumnos con las herramientas necesarias para acceder a los conocimientos y datos disponibles y poder explorarlos en este mundo globalizado. Por esta razón, la profesión docente está cambiando desde un enfoque centrado en el profesor y basado en clases tradicionales, hacia una formación centrada en el alumno dentro de un entorno interactivo de aprendizaje. La Universidad Autónoma de Nuevo León (UANL) enfrenta las demandas sociales para integrarse al mundo de la globalización y plasma sus fines en el Modelo Educativo 2015 (UANL, 2015). En este documento "las tecnologías de la información y la comunicación constituyen uno de los factores que han acelerado y modificado los procesos de manejo de la información y comunicación" (UANL, 2015, p. 18). Se establecen implicaciones como: "cambios profundos en la pedagogía, nuevos enfoques y otras formas de aprendizaje y enseñanza, transformación del papel tradicional del profesor y del estudiante, mejoramiento de la calidad de educación, dinamización del proceso educativo, desarrollo de alternativas pedagógicas y metodológicas, y aplicación en los procesos de formación de profesores, con el perfil idóneo para el desarrollo de las funciones universitarias" (UANL, 2015, p. 18).

Además, se propone la capacitación a profesores en el uso de las nuevas tecnologías para incorporarlas a su práctica educativa. También se señala que como consecuencia los profesores se sensibilizan acerca de la importancia de desarrollar

Corresponding author, Universidad Autónoma de Nuevo León, México, https://orcid.org/0000-0002-3849-5809, Email: elizalv@yahoo.com.mx 
sus tareas con un enfoque que privilegia más el aprendizaje que la enseñanza, cambiando su concepción y rol de trabajo, es decir se cita un modelo de enseñanza-aprendizaje integral basado en el alumno y en el aprendizaje significativo. Asimismo, se distinguen diferentes roles de los docentes universitarios tales como asesor, facilitador, motivador y asesor (UANL, 2015, p.19). Cabe destacar que, en el perfil del docente, el profesor debe manejar las tecnologías de la información y la comunicación como herramienta para el acceso a la información y su transformación en conocimiento, así como para el aprendizaje y trabajo colaborativo con técnicas de vanguardia que le permitan su participación constructiva en la sociedad (UANL, 2018, p. 20).

Por lo tanto, en el Plan de Desarrollo Institucional 2019-2030, UANL Visión 2030, se expresan los retos que enfrenta la UANL como el aseguramiento de la aplicación de los modelos educativos y académicos en la impartición de todos sus programas educativos, además de la búsqueda de formación y certificación de los profesores (UANL, 2019). De ahí que todo docente universitario requiere conocer y utilizar las tecnologías. Sin embargo, habría que investigar el porcentaje de los docentes universitarios que sí conocen y utilizan las TIC en su labor educativa. Estos datos podrían servir como punto de partida para la planeación de un programa de capacitación y desarrollo de dichas competencias y conocimientos en el cuerpo docente. Pues muchos de ellos no las utilizan o creen que las TIC les resolverán los problemas de enseñanza-aprendizaje o bien, hacen uso de presentaciones en Power Point sin una estrategia planeada con anticipación.

\section{EL CAMBIO TECNO-PEDAGÓGICO}

"Las asociaciones profesionales, países, estados o universidades a menudo colaboran con la comunidad educativa para establecer estándares y lineamientos que describan la habilidad, el conocimiento y la motivación que los candidatos deben de haber alcanzado en el campo de las TICs al terminar su formación como docentes" (Khvilon, 2004, p. 55), ejemplo de esto es la UANL. Es necesario que tanto los futuros docentes como los docentes en actividad sepan utilizar las herramientas tecnológicas para aplicarlas en el proceso de enseñanza-aprendizaje tanto en la enseñanza de una lengua extranjera como de contenido, tal como lo comentan Cuevas y García (2014) y que además, señalan los beneficios de incorporar las TICs, entre estos: el mejoramiento del currículo mediante la incorporación de nuevos contenidos, la resolución de problemas de reflexión y retroalimentación, la construcción de comunidades y espacios de aprendizajes, el desarrollo profesional de los docentes, la autonomía y nuevos roles tanto del alumno como del docente. Hay estudios que muestran que los profesores y alumnos utilizan más las computadoras en el hogar que en la escuela mayormente para fines personales (De Alba y Glazman, 2009). Aunque un factor de este uso puede ser la falta de infraestructura en las instituciones y que las TIC no surgieron para resolver problemas educativos. Es decir, no se realizó un proyecto previo respecto al diseño de la enseñanza y el aprendizaje, ni mucho menos de la formación docente para incluir las TIC (3).

Salas (2011) señala que el proceso de incorporación de las TIC se ha centrado en el aprendizaje y no en la mediación docente, se asume, que se ha dado un cambio en cuanto a las tecnologías mas no en la conceptualización del uso educativo de las mismas y en los métodos pedagógicos y herramientas de aprendizaje, por eso es importante que la UANL haya incluido en su Plan de Desarrollo Institucional 2019-2030 los nuevos roles que los docentes deberán asumir. Para llegar a ello, debe producirse un cambio en la concepción tradicional del proceso de aprendizaje y una nueva comprensión acerca de cómo las TIC pueden ayudar a crear nuevos entornos de aprendizaje en los que los alumnos se sientan más motivados y comprometidos, asuman mayores responsabilidades sobre su propio aprendizaje y puedan construir con mayor independencia sus propios conocimientos. No obstante, la reflexión y el cambio de las creencias y posiciones educativas de los docentes no se dan con rapidez, al respecto Gregoire (2003) y Dole y Sinatra (1998) indican que las creencias son difíciles de cambiar y que se requiere que el profesor esté involucrado activamente en el proceso sistemático del conocimiento. Se requiere profundizar en el asunto, y desarrollar una reflexión metacognitiva, pues los profesores también tienen que aprender a aprender con respecto a la computadora, al internet, y a las TIC. Además, si los docentes carecen de una formación pedagógica profesional, entonces tendrían que enfrentarse a una doble tarea: a una formación pedagógica y a una formación tecnológica. En el caso de la UANL, los profesores de lengua extranjera no sólo tienen esa tarea tecno-pedagógica, ellos también deben actualizarse y certificarse en cuestiones de la lengua.

Por otro lado, estas tareas que son parte del desarrollo profesional de los docentes estarían enmarcadas en el ámbito de la educación para adultos. Es decir, los adultos poseen esquemas mentales formados a través de los años y experiencias, y al enfrentarse a una formación tecno-pedagógica se espera que estos esquemas se transformen. Uno de los modelos que enfatiza esta idea de transformación es la teoría del aprendizaje transformativo que en inglés se conoce como transformative learning. Esta teoría "describe cómo los adultos integran nueva información, perspectivas, o práctica en sus visiones de mundo al comprometerse en el aprendizaje. Cuando los aprendices se comprometen con las oportunidades para reflexionar sobre el significado de lo que están aprendiendo, pueden comprometerse en la evaluación de sus valores familiares, creencias y supuestos" (King, 2004, p. 155, citado en Espejo y González, 2015). Entonces, para que las creencias y formas de actuar de los docentes hacia nuevas formas de enseñar y la tecnología se transformen, se requiere de reflexión.

Este cambio de paradigma en la concepción del 
proceso de aprendizaje tiene sus desventajas, por ejemplo, los maestros tienen dificultad con enseñar a los alumnos a aprender en los entornos mixtos (virtuales y presenciales), debido a que las instrucciones son complicadas, hay alumnos que prefieren ver cara a cara al maestro y poder relacionarse más con los compañeros. Casarini (citada en De Alba y Glazman, 2009) menciona que muchas veces los programas de cursos presenciales son subidos a la plataforma tal y como fueron diseñados para una clase en el aula. Entonces, el alumno tiene que enfrentarse a actividades difíciles de comprender y realizar con las TIC. Es ineludible más organización y especificidad en el diseño de los cursos en donde se utilizan las TIC, pero sobre todo en los contextos virtuales. Los maestros tienen que convertirse en escritores y desarrollar más sus competencias comunicativas a través de la escritura para poder dirigir al alumno. Casarini, (citada en De Alba y Glazman, 2009) indica que la autonomía de los aprendices en la virtualidad y el vínculo con el profesor es un tema importante, ya que es el mismo alumno quien se convierte en responsable de su propio aprendizaje, se autodirije. La autora añade que la autodirección tiene dos tipos de dimensiones diferenciadas: la dimensión de aprendizaje autodirijido, donde asume su responsabilidad en la planeación, elaboración y evaluación del proceso de aprendizaje y, la dimensión de autodirección del estudiante donde él tiene interés por hacerse cargo de su propio aprendizaje. El rol del profesor (ahora facilitador, tutor, moderador, monitor, guía, consejero, asesor, instructor, administrador) es diseñar experiencias de aprendizaje de múltiples tipos. Por ello, es importante considerar la autodirección en las decisiones del diseño didáctico de los cursos. Asimismo, Casarini (2007) recomienda que se debe tomar en cuenta la relación entre maestro-alumno ya que ésta depende de la disposición y tiempo del alumno para acercarse al profesor y de la disposición del profesor para tutorear, además de la cantidad de alumnos que atender.

Recordemos que los profesores y diseñadores deben estar al tanto de la planeación, de la metodología y tratar de integrar materiales didácticos multimedia para que se dé una buena comunicación entre alumnos y maestros, para que no estén aislados y trabajen colaborativamente; por lo tanto la tecnología es una herramienta y pocas veces un fin en sí (Casarini, 2007). Se trata de incorporar actividades valiosas y no dejar que la didáctica silenciosa se lleve a cabo, pues muchos profesores dejan hablar al material audiovisual sin alguna estrategia diseñada para la actividad con dicho material (Litwin, 2005), principalmente en clases de una lengua extranjera en donde se requiere que el alumno tenga un rol participativo, activo e intercativo.

\section{PARADIGMA EDUCATIVO}

Khvilon (2004) señala que tradicionalmente el profesor es el experto y quien transmite la información a los alumnos. Este paradigma educativo tradicional se caracteriza por presentar las siguientes concepciones acerca del aprendizaje:

\section{- Aprender es difícil \\ - El aprendizaje se basa en un modelo centrado en el déficit. \\ - El aprendizaje es un proceso individual/solitario. \\ - El aprendizaje es un proceso lineal.}

El nuevo paradigma educativo, según Khvilon (2004), abarca los siguientes conceptos en contraste con el paradigma tradicional de enseñanza-aprendizaje:

- El aprendizaje es un proceso natural porque el cerebro tiende naturalmente a aprender, aunque no todos aprenden de la misma manera. Existen distintos estilos de aprendizaje, y personalidades, que deben tomarse en cuenta al momento de diseñar las experiencias de aprendizaje para los alumnos individuales.

- El aprendizaje es un proceso social. Como señalaba Vygotsky hace tiempo, los alumnos aprenden mejor en colaboración con sus pares, profesores, padres y otros, cuando se encuentran involucrados de forma activa en tareas significativas e interesantes. Las TIC brindan oportunidades a docentes y alumnos de colaborar con otros individuos en cualquier parte del país o del mundo. También ofrecen nuevas herramientas para apoyar este aprendizaje colaborativo tanto dentro del salón de clase como conectados a la Red.

- El aprendizaje es un proceso activo, no pasivo. Para que los alumnos alcancen niveles óptimos de competencia, deben ser motivados a involucrarse de forma activa en el proceso de aprendizaje, en actividades que incluyan resolver problemas reales, producir trabajos escritos originales, realizar proyectos de investigación científica, y dialogar con otros acerca de temas importantes, entre otras. Machado, (2015, p. 2) recalca que el rol del profesor es tan activo como el de los estudiantes.

- El aprendizaje puede ser tanto lineal como no lineal. La mente es un maravilloso procesador, que puede prestar atención y procesar muchos tipos de información simultáneamente. La teoría e investigación cognitiva ve el aprendizaje como una reorganización de las estructuras de conocimiento, las cuales se guardan en la memoria semántica como esquemas o mapas cognitivos. Padilla (2004) indica que la memoria tiene estructura, a la vez que permite mostrar cómo el conocimiento está conectado en la memoria. Los alumnos "aprenden" al ampliar, combinar y reacomodar un grupo de mapas cognitivos, que muchas veces se superponen o están interconectados por medio de una compleja red de asociaciones.

- El aprendizaje es integrado y contextualizado. La información que se presenta de un modo global es más fácil de asimilarse que la que se presenta como una secuencia de unidades de información. Además, permite que los alumnos puedan ver la relación entre los distintos elementos y puedan crear conexiones 
entre ellos sin ayuda. El rol del docente es ayudarlos de diversas maneras a realizar estas conexiones y a integrar el conocimiento. Perin (2011) subraya la importancia de facilitar el aprendizaje a través de situaciones o contexto tanto en clases de inglés como de contenido, es decir de cualquier asignatura.

- El aprendizaje se evalúa según los productos del proceso, la forma en que se completan las tareas y la resolución de problemas reales, tanto por parte de cada estudiante como del grupo. En lugar de evaluar al alumno únicamente por medio de pruebas escritas, la evaluación se realiza basándose en carpetas de trabajo (portafolios) donde el alumno muestra su desempeño en los trabajos realizados en equipo o de forma individual. En el mundo actual se requiere que los estudiantes puedan trabajar en equipo (interactivamente), pensar de forma crítica y creativa y reflexionar acerca de su propio proceso de aprendizaje, tal es el caso de la UANL, en donde se proponen nuevas formas de evaluar en sus modelos educativos y académicos (UANL, 2019), cuestión que favorece la evaluación del proceso de aprendizaje en lenguas extranjeras.

Pero, ¿y qué sucede con los estudiantes? ¿Qué se espera de ellos en este ambiente de las TIC? Los alumnos deberán insertarse en un entorno rico en información, ser capaces de analizar y tomar decisiones, de dominar nuevos ámbitos del conocimiento en una sociedad cada vez más tecnológica. Deberán convertirse en estudiantes de por vida, colaborando con otros individuos para realizar tareas complejas y utilizando de modo efectivo los diferentes sistemas de representación y comunicación de conocimiento.

La conversación es la base social de intercambio comunicativo, pues sólo no se avanza igual y de acuerdo a Beaudin (citado en Bautista, 2008, p.147), "los estudiantes se concentran mejor en discusiones temáticas con un fuerte contenido conceptual cuando se establecen buenas preguntas, cuidadosamente diseñadas, se proveen lineamientos para la discusión". Además, esta comunicación se convierte en una conversación didáctica guiada por ser orientada al aprendizaje y si se utiliza un estilo sencillo y amistoso invitando al alumno a considerar sus aciertos y corregir errores de una manera pertinente, los estudiantes tienen la oportunidad de expresarse más libremente y adquirir confianza con el profesor. Por lo tanto, los estudiantes deberán enfrentarse al aprendizaje centrado en el alumno y respetar los nuevos roles de tanto los docentes como de ellos mismos. Hoy el profesor se ubica al lado del estudiante, por lo que se necesita una planeación anticipada y detallada (Bautista, 2008).

\section{TEORÍAS QUE RESPALDAN EL PARADIGMA EDUCATIVO}

¿Qué teorías respaldan la nueva concepción acerca del proceso de aprendizaje? De acuerdo con Khvilon (2004), las formas de percibir el proceso de aprendizaje y el cambio hacia un aprendizaje centrado en el alumno, se basan en investigaciones sobre el aprendizaje cognitivo y la convergencia de diversas teorías acerca de la naturaleza y el contexto del aprendizaje. Este autor, plantea que algunas de las teorías más importantes son: la teoría sociocultural (basada en las intersubjetividades y la Zona de Desarrollo Próximo de Vygotsky), la teoría constructivista, el aprendizaje auto-regulado, la cognición situada, el aprendizaje cognitivo, el aprendizaje basado en la resolución de problemas (del Grupo de Cognición y Tecnología de Vanderbilt, $C T G V$ ), la teoría de la flexibilidad cognitiva y la cognición distribuida. Estas teorías mencionan que los estudiantes son agentes activos que buscan y construyen conocimiento con un propósito, dentro de un contexto significativo. El alumno interactúa con otros alumnos, con el docente, con los recursos de información y con la tecnología. El alumno se involucra en tareas reales que se llevan a cabo en contextos reales, utilizando herramientas que le sean de útiles, y es evaluado de acuerdo a su desempeño en términos realistas. Al alumno se le provee con un andamiaje de apoyo para desarrollar sus conocimientos y habilidades. A su vez, provee un entorno rico en colaboración, lo que permite al alumno considerar múltiples perspectivas al abordar ciertos temas y resolver problemas, y brinda oportunidades para que el alumno pueda reflexionar sobre su propio aprendizaje (Khvilon, 2004).

Aunque estas teorías señalan a los alumnos como agentes activos podría ser que algunos de ellos se encierren en un mundo digital, podrían poseer una dependencia al internet y a las nuevas tecnologías, es decir, podrían padecer tecnofilia (Hervás, López, Real y Fernández, 2016). No habría una interacción con otros alumnos o docentes por parte de este tipo de alumnos. Es aquí donde el docente juega un papel imperante pues es necesario que éste recuerde las ventajas de la tecnofilia en el proceso de aprendizaje. Aunque puede darse el caso que algún docente prefiera seguir trabajando con métodos tradicionales y rechacen cualquier tipo de tecnología (tecnofobia), también puede haber un docente que se sienta identificado con la tecnología y la incorpore en su praxis diaria. Este docente podría ayudar a los alumnos en el control del uso de las tecnologías a través de estrategias de enseñanza y aprendizaje aplicadas a su planeación de clase.

\section{REFLEXIÓN}

Aunque este nuevo entorno de aprendizaje puede crearse sin hacer uso de la tecnología, es claro que las TIC constituyen una herramienta decisiva para ayudar a los estudiantes a acceder a muchos recursos de conocimiento, a colaborar con otros compañeros, consultar a expertos, compartir conocimiento y resolver problemas complejos utilizando herramientas cognitivas. En consecuencia, se requiere concentrarse en una actualización técnico-pedagógica para que los profesores desarrollen habilidades didácticas relevantes y proporcionen en los alumnos un aprendizaje significativo tal y como Ausbel lo plantea; un diseño de contenidos con sentido para los estudiantes 
(Bautista, 2008). De esta forma las TIC se integren dentro de un contexto, cuestión importante en las clases de lengua extranjera al realizarse actividades en donde los estudiantes puedan practicar el idioma en situaciones cercanas a la realidad. Aunque para ello es necesario enseñar a los docentes a utilizar las herramientas básicas de la computadora. Conocer el sistema operativo tradicional, el procesador de texto (Word), las hojas de cálculo (Excel), Power Point, las bases de datos y las herramientas de telecomunicación, no es suficiente. Como en toda profesión, existe un nivel de manejo que supera el conocimiento común acerca del uso de una computadora y la mayoría de los docentes somos inmigrantes digitales, es decir, nacimos en una cultura anterior a las TIC (Coll y Monereo, 2008).

Este conocimiento más específico incluye aprender a utilizar la tecnología para motivar el crecimiento educativo de los alumnos de la UANL, en otras palabras, sacar el máximo partido a las tecnologías con el objetivo de desarrollar el pensamiento crítico en los alumnos y romper la brecha digital existente entre alumnos y maestros y así implementar el Plan de Desarrollo Institucional 2019-2030, UANL Visión 2030. Los docentes deben familiarizarse con los usos de la tecnología, ya que se ven obligados a utilizarla dentro de sus propios cursos y prácticas docentes, como lo señalan Viñals y Cuenca (2016), se requiere ser competente en la era digital, renovarse y "adaptar las metodologías de enseñanza al nuevo entorno" (113). Para luego investigar usos creativos de la tecnología e implementarlos en su propia actividad docente. La tecnología puede utilizarse para apoyar formas tradicionales de educación, así como para transformar el aprendizaje en donde se motive al alumno a hacer uso crítico de la tecnología. Una presentación en Power Point, por ejemplo, puede mejorar una clase tradicional, pero no necesariamente transformar la experiencia de aprendizaje. Por otra parte, el uso de herramientas multimedia para enseñar ciertos temas que han sido abordados anteriormente, es un ejemplo de cómo la tecnología puede transformar la experiencia de aprendizaje. Los alumnos deben experimentar ambos tipos de uso de la tecnología dentro de sus cursos. Sin embargo, el uso más prometedor de la tecnología en la educación es como apoyo a formas más innovadoras y creativas de enseñanza y aprendizaje.

Villegas y Alvarado (2017) mencionan que una de las formas innovadoras que es el uso de la gamificación, considerada como herramienta educativa, a fin de promover conductas y hábitos que acrecienten la adquisición de conocimientos y que conduzcan a los estudiantes al desarrollo de competencias, mejorando su rendimiento académico, y sobretodo aumentando su motivación tanto intrínseca como extrínseca, pues hoy en día, los estudiantes dedican gran parte de su tiempo en el uso de las redes sociales, aplicaciones digitales y videojuegos, entre otros simuladores.
Por todo lo expuesto arriba de estas líneas, la formación docente inicial y continua debe incluir una actualización constante en los usos de las TICs, puesto que ellas mismas están en un estado constante de evolución. Los docentes deben actualizar sus conocimientos y habilidades, revisando los planes de estudio continuamente. Ese desarrollo profesional y personal debe acompañarse por un desarrollo organizacional en las escuelas, centros de formación y universidades. Hay instituciones que han puesto en marcha proyectos de innovación educativa en donde incluyen el uso de las TIC al mismo tiempo que van capacitando a su personal docente y técnico. Lo óptimo sería que los docentes recibieran una preparación antes de comenzar a dar clases en las escuelas; o bien que se llevara a cabo la tutoría a profesores noveles por parte de un docente con experiencia en el uso de las TIC (tutorías de pares), aunque es una estrategia de aprendizaje en la que estudiantes orientan a sus compañeros (Barbosa y Barbosa, 2019), ésta puede realizarse entre la comunidad docente y producir beneficios para los profesores. Esto no sólo aumenta el potencial de investigación del docente tutor, sino que permite al tutorado contar con un modelo que podría facilitarle la transición hacia el ejercicio de su profesión. Así se tendría una comunidad de aprendizaje en la que se brinda apoyo a otros colegas aumentando la motivación, la autoestima y creando un mejor ambiente de trabajo. Cabe mencionar que la formación tecno-pedagógica que se ofrecería a los docentes nunca debe ser impuesta, es recomendable ofrecerla sin ninguna represalia. Es preferible que el propio profesor esté convencido de las necesidades de una formación continua. Por otro lado es trascendental reconocer la importancia de capacitar a los docentes desde el comienzo de sus carreras o de su inicio como profesor y además, de elaborar un programa sistemático de educación continua para el cuerpo docente de cada institución de educación superior, con el fin de mantenerse al corriente con las innovaciones en los TICs y en la psicopedagogía.

\section{REFERENCIAS}

[1] Barbosa, J y Barbosa, Ch. (2019). La tutoría entre pares: Una mirada al contexto universitario en Latinoamérica. Revista Espacios, 5(5), p. 30. http://www.revistaespacios.com/a19v40n15/a19v40n15p30.pdf

[2] Bautista, R. A. (2008). Educación y tecnologías de la información y la comunicación. Plaza y Valdez editores.

[3] Casarini, M. (2007). La Interacción y el diseño de los aprendizajes en contextos virtuales en Tecnología Educativa: en un modelo de educación a distancia centrado en la persona. Limusa.

[4] Coll, C. y Monereo, C. (2008). Psicología de la educación virtual. Morata.

[5] Cuevas, F. y García, J. (2014). Las TIC en la formación docente. Congreso Iberoamericano de Ciencia, Tecnología, Educación $e$ Innovación. Argentina. www.oei.es , historico > congreso2014 , 21 memorias 2014

[6] De Alba, A. y Glazman R. (2009) Coordinadoras. ¿Qué dice la investigación educativa? COMIE.

[7] Dole y Sinatra (1998). Dole, J., \& Sinatra, G. (1998). Reconceptualizing change in the cognitive construction of knowledge. Educational Psychologist, 32, 109-128. 
[8] Espejo, R. y González, J. M. (2015). Aprendizaje transformativo y programas de investigación en el desarrollo docente universitario. Revista de Docencia Universitaria,13(3), pp.309-330.

[9] Gregoire (2003). Gregoire, M. (2003). Is it a challenge or a threat? A dual-process model of teachers' cognition and appraisal processes during conceptual change. Educational Psychology Review, 15, 147179.

[10] Hervás, C., López, E., Real, S., \& Fernández, E. (2016). Tecnofobia: competencias, actitudes y formación del alumnado del Grado en Educación. International Journal of Educational Research and Innovation (IJERI), 6, pp. 83-94.

[11] Khvilon, E. (2004) Coordinador. Las tecnologías de la información y la comunicación en la formación docente Trilce: Paris, Francia. www.http://unesdoc.unesco.org/images/0012/001295/129533s.pdf

[12] Litwin, E. (2005). Tecnologías Educativas en tiempos de internet. Amorrortuo Editores.

[13] Machado, E. M. (2015). Los paradigmas educativos. Revista Arista Digital.,52,1-5.

http://www.afapna.es/web/aristadigital/archivos_revista/2015_febrero_ 7.pdf

[14] Padilla, V. M. (2004). Innovación en la medición cognitiva del aprendizaje significativo en una plataforma de internet: relación con estilos cognitivos y de aprendizaje. Tesis de doctorado, Universidad Autónoma de Nuevo León.

[15] Perin, D. (2011). Facilitating student learning through contextualization. Community College Research Center. Columbia University.

USA https://ccrc.tc.columbia.edu/media/k2/attachments/facilitating-learningcontextualization-working-paper.pdf

[16] Salas, F. (2011). La integración del as TIC en la docencia en la UCR: estudio desde un enfoque sistémico-complejo del personal docente que se certificó en el curso Educar para el futuro, versión universitaria en el periodo 2003-2006. Tesis doctoral Universidad de Costa Rica. www.ojocientifico.com

[17] Universidad Autónoma de Nuevo León (2015). Modelo Educativo. UANL. Ciudad Universitaria, Nuevo León: UANL.

[18] Universidad Autónoma de Nuevo León (2018). Modelo Académico. Nivel Medio Superior. UANL. Ciudad Universitaria, Nuevo León: UANL.

[19] Universidad Autónoma de Nuevo León (2019). Plan de Desarrollo Institucional 2019-2030. UANL Visión 2030. Ciudad Universitaria, Nuevo León: UANL

[20] Villegas, B. y Alvarado, E. (2017. Gamification: a didactic strategy in higher education. EDULEARN17 Proceedings, pp. 6761-6771 https://library.iated.org/view/VILLEGASAGUILERA2017GAM

[21] Viñals, A. y Cuenca, J. (2016). El rol del docente en la era digital. Revista Interuniversitaria de Formación del Profesorado, 3(2), pp. 103-114 https://www.redalyc.org/pdf/274/27447325008.pdf 